using the Paykel's Interview for Recent Life Events. Pain was assessed using a visual analogue scale (VAS). The Fibromyalgia Impact Questionnaire (FIQ) was also used.

Results: Seventy-seven patients were originally screened, but seven were excluded because of current depressive episode or having a ZSDS of $\geq 60$ or categorized as minimizers of childhood maltreatment at CTQ. The final analysis therefore involved 70 patients, all Caucasians: 30 with PFM and 40 with AR+FM. All patients with PFM and $38(95 \%)$ of the 40 with AR+FM were treated for FM symptoms (antidepressants, pregabalin). The lifetime rates of MDD were significantly higher in PFM vs AR+FM $(76.7 \%$ and $40 \%$ respectively, $p$ $=0.003)$, as well as the rates of PD (50\% and $15 \%$ respectively, $p=0.003)$, whereas there was no difference in PTSD rates. The PFM patients reported significantly higher levels of physical $(p=0.020)$ and sexual abuse $(p=0.011)$ and physical neglect $(p<0.001)$, whereas there was no between-group difference in the levels of emotional abuse $(p=0.912)$ and neglect $(p=0.542)$; consistently, the proportion of sexually abused $(p=0.005)$ or physically neglected patients was also higher in the PFM group $(p=0.023)$. The rates of emotional neglect were high in both groups, without any significant difference between them. The vast majority of $A R+F M$ patients $(90 \%)$ said that only event occurring in the year preceding the onset of FM was RA, whereas the PFM patients mainly reported non-physical events $(36 \%$, particularly the ending of a relationship, or working or financial problems) or no event at all $(40 \%),(p<0.001)$. Binary logistic regression used to identify the factors predicting association of $\mathrm{PFM} / \mathrm{AR}+\mathrm{FM}$ status, showed an association with lifetime major depression, life events preceding the development of FM, and BMI $(p<0.05$ at all).

Conclusion: PFM and SFM differ in psychiatric co-morbidities and environmental adversities, suggesting that the putative common pathogenetic condition of CS may develop through different pathways.

References:

[1] McWilliams DF, Walsh DA. BMC Musculoskelet Disord 2016; 17, 337.

Disclosure of Interests: : None declared

DOI: 10.1136/annrheumdis-2020-eular.3894

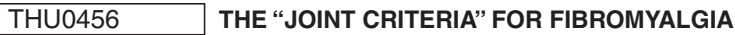 DIAGNOSIS IN RHEUMATOID ARTHRITIS PATIENTS: RELIABILITY COMPARED TO THE 2010 ACR CLASSIFICATION CRITERIA FOR FIBROMYALGIA}

L. J. Ghib ${ }^{1}$, A. Barcic ${ }^{1}$, A. D. Bilous ${ }^{2}$, I. Cozma ${ }^{2}$, M. M. Tamas ${ }^{1,3}$, I. Filipescu $^{1,3}$

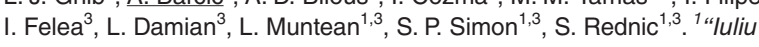
Hatieganu" University of Medicine and Pharmacy, Rheumatology, Cluj-Napoca, Romania; ${ }^{2}$ Rehabilitation Clinical Hospital, Cluj-Napoca, Romania; ${ }^{3}$ Emergency Clinical County Hospital Cluj, Rheumatology, Cluj-Napoca, Romania

Background: A significant proportion of rheumatoid arthritis (RA) patients have concomitant fibromyalgia (FM) (1). Associated FM diagnosis in RA patients can determine worse treatment outcomes compared to patients without FM (1). A difference between tender joint count (TJC) and swollen joint count (SJC) $\geq 7$, also named the "joint criteria" was proposed as being diagnostic for FM in patients with RA. The "joint criteria" were validated against the 1990 ACR Classification Criteria for FM and are easy to apply to patients with RA (2). Since then, the 2010 ACR Classification criteria for FM, which include somatic symptoms besides pain sensitivity, were developed and validated.

Objectives: We aimed to determine the reliability of the joint criteria for fibromyalgia in RA compared to the ACR 2010 Classification Criteria for FM and to compare RA patients diagnosed with FM (FRA) to those without FM in terms of clinical variables.

Methods: We performed a cross-sectional study on RA patients who presented in our department during a 3 months period. Tender joint count (TJC), swollen joint count (SJC), patient global assessment of disease activity (PGA) were determined. DAS28 scores were calculated using CRP. We applied the 2010 ACR Classification Criteria and the joint criteria for FM diagnosis. Kappa agreement coefficient was used to determine the reliability of the joint criteria against the 2010 ACR Classification Criteria for FM in patients with RA. Differences between groups were assessed using Mann-Whitney $U$ test for numerical data or Chi square test for ordinal data.

Results: We included 100 consecutive RA patients, $84 \%$ female, with a mean age of $57.3(12)$ years and mean disease duration of 14(9) years. Twenty-four patients (24\%) had associated FM according to the ACR 2010 Classification Criteria and $22(22 \%)$ patients satisfied the joint criteria for associated FM. The level of agreement between the joint criteria and the ACR 2010 classification criteria for FM was kappa=0.66, $p<0.001$, with a sensitivity of $70 \%$ and a specificity of $93 \%$. FRA patients had similar demographic and disease characteristics compared to RA patients. Patients with FRA according to the joint criteria had significantly higher PGA, DAS28 and $H A Q$ scores, but similar CRP values and SJC compared to RA patients (Table 1).

Table 1 Demographic and clinical data of FRA and RA patients

\begin{tabular}{lccc}
\hline Variable & FRA $\mathbf{n}=\mathbf{2 2}$ & RA $\mathbf{n}=\mathbf{7 8}$ & p-value \\
\hline Age (years) & $60(10.7)$ & $59(12.2)$ & 0.093 \\
Disease Duration (years) & $13.3(13)$ & $12.2(7.5)$ & 0.589 \\
ACPA seropositivity(\%) & 69 & 55 & 0.1 \\
SJC & $2(4)$ & $2(4)$ & 0.7 \\
CRP (g/dl) & $12.8(14.2)$ & $8.1(13.7)$ & 0.06 \\
DAS28CRP & $4(1.7)$ & $3.5(1.2)$ & 0.009 \\
HAQ & $1.75(0.5)$ & $1(0.7)$ & $<0.001$ \\
PGA (mm) & $70(11)$ & $44(23)$ & $<0.001$ \\
\hline
\end{tabular}

Data are expressed as mean (SD) or median (IQR)

FRA- Fibromyalgic Rheumatoid Arthritis; RA- Rheumatoid Arthritis;

ACPA- Anti- citrullinated Protein Antibodies; CRP- C-reactive Protein; SJC- Swollen Joint Count;

DAS28CRP- Disease Activity Score; HAQ- Health Assessment Questionnaire; PGA- Patient Global Assessment

Conclusion: The joint criteria are diagnostic for FM in RA patients with moderate reliability compared to the ACR 2010 Classification criteria. When diagnosed with the joint criteria, FRA patients have higher disease activity scores despite having similar clinical and laboratory inflammatory markers compared to RA patients. References:

[1] Wolfe F, Michaud K. Severe rheumatoid arthritis (RA), worse outcomes, comorbid illness, and sociodemographic disadvantage characterize RA patients with fibromyalgia. J Rheumatol. 2004;31(4):695-700.

[2] Pollard LC, Kingsley GH, Choy EH, Scott DL. Fibromyalgic rheumatoid arthritis and disease assessment. Rheumatology 2010;49(5):924-8.

Disclosure of Interests: : None declared

DOI: 10.1136/annrheumdis-2020-eular.3285

\section{THU0457 LONGITUDINAL ASSOCIATION OF SEDENTARY TIME AND PHYSICAL ACTIVITY WITH SLEEP QUALITY IN WOMEN WITH FIBROMYALGIA:THE AL-ÁNDALUS PROJECT}

M. Borges Cosic ${ }^{1}$, P. Acosta-Manzano², B. Gavilán Carrera², F. EstévezLópez $^{3}$, V. A. Aparicio ${ }^{4}$, V. Segura-Jiménez ${ }^{5}$, M. Delgado-Fernández ${ }^{2}$ ${ }^{1}$ University of Granada, Faculty of Sport Sciences, Department of Physical Education and Sport, Granada, Spain; ${ }^{1}$ University of Granada, Faculty of Sport Sciences, Department of Physical Education and Sport, Granada, Spain; ${ }^{3}$ Erasmus MC University Medical Center, Department of Child and Adolescent Psychiatry/Psychology, Rotterdam, Netherlands; ${ }^{4}$ University of Granada, Faculty of Pharmacy, Department of Physiology, Granada, Spain; ${ }^{5}$ University of Cádiz, Faculty of Physical Education, Department of Physical Education, Cádiz, Spain

Background: Sleep disturbances are common in fibromyalgia, and influences quality of life. Recent literature has suggested that non-pharmacological treatments (e.g., physical exercise and cognitive behavioural therapy) may help to improve sleep quality (SQ) and the management of fibromyalgia ${ }^{1}$. In this regard sedentary time (ST) and physical activity (PA) intensity levels could play a role on $S Q$ in this population ${ }^{2}$. However, evidence is scarce and mainly based on cross-sectional data.

Objectives: This study aimed to examine the longitudinal associations (2and 5-year follow-up) of ST and PA intensity levels with SQ in women with fibromyalgia.

Methods: In this prospective cohort study, women diagnosed with fibromyalgia (age: $51.4 \pm 7.6$ years) with completed data were included at baseline $(n=409)$, at 2-year follow-up $(n=214)$ and at 5-year follow-up $(n=218)$. Sedentary time and PA intensity levels (light and moderate-to-vigorous [MVPA]) were assessed using triaxial accelerometers worn for consecutive 7 days. The percentage of time spent in different behaviours was calculated (e.g., (ST/accelerometer wear time) $\times 100$ ). The SQ global score was calculated as a sum of all components (score 